\title{
Rectal Meckel's diverticulum
}
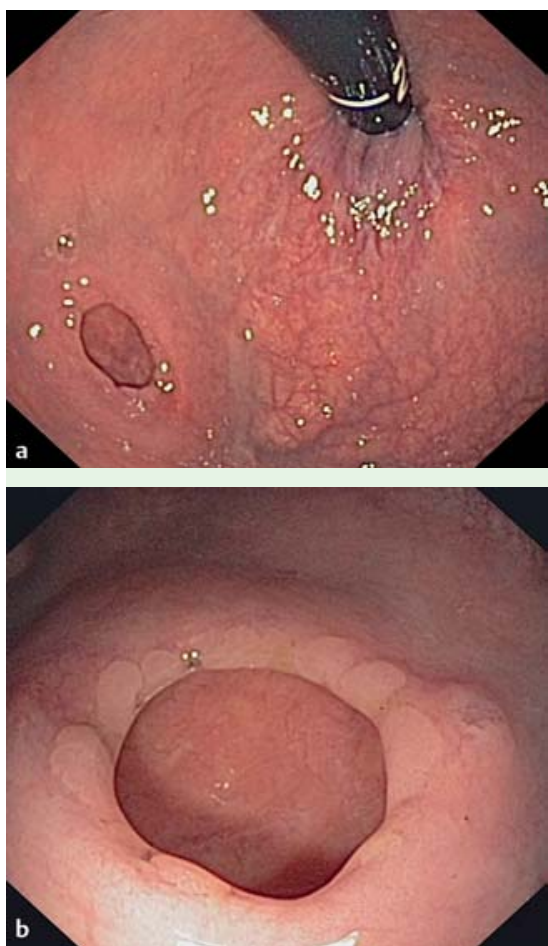

Fig. 1 a, b Retroflexed endoscopic views of the rectal Meckel's diverticulum.

A 39-year-old woman presented for colon cancer screening colonoscopy. She had no abdominal complaints. The entire colonoscopy was normal, except that the standard retroflexed view in the rectum demonstrated a 2-cm diverticulum without raised margins $5 \mathrm{~cm}$ from the anal verge ( $\bullet$ Fig. 1 a, b).

Endoscopically the mucosa appeared fairly edematous, but without malignant characteristics. The histological examination of the biopsies obtained revealed rectal mucosa abutting ectopic gastric mucosa without Helicobacter pylori (๑ Fig. 2).

The technetium Tc $99 \mathrm{~m}$ pertechnetate scan was negative. Follow-up sigmoidoscopy performed 3 months later showed an unchanged diverticulum. Again, histological examination demonstrated ectopic gastric mucosa. As the patient remained asymptomatic, to date no surgical

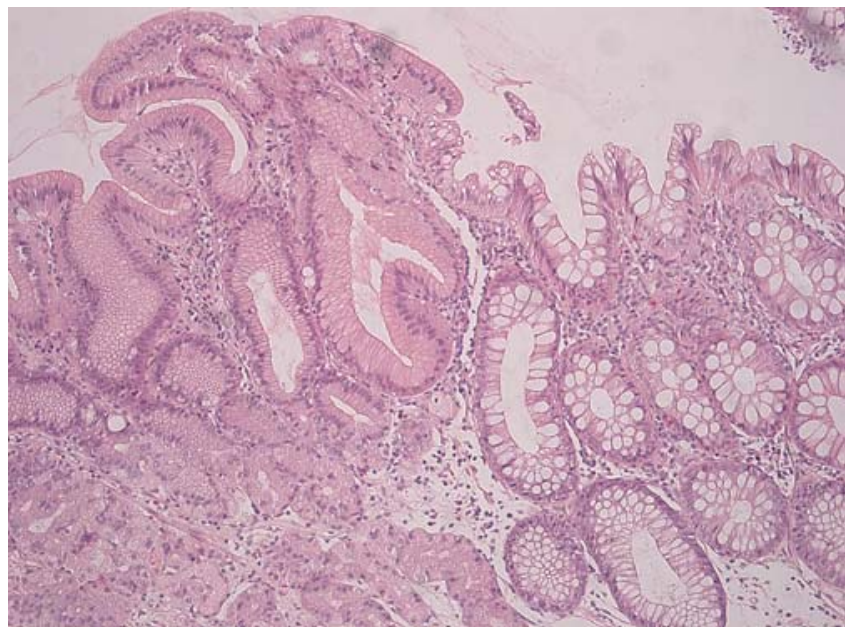

Fig. 2 Histological section shows rectal mucosa abutting ectopic gastric mucosa (hematoxylin and eosin staining, $\times 100$ )

resection or further endoscopic screening has been performed.

Heterotopic gastric mucosa can be observed anywhere along the human gastrointestinal tract. It is rarely seen in the rectum: only about 30 cases have been reported since the first description in 1939 [1]. In the clinical setting, gastric heterotopia typically presents as either an ulcerative lesion or abnormal mucosa in the rectum [2]. The origin of gastric mucosa in the rectum is believed to be failure of developmental descent of the embryonic hindgut. The occurrence of gastric heterotopia within a hindgut developmental anomaly such as rectal duplication is exceptional [3]. In clinical practice, a so-called rectal Meckel's diverticulum may present with acute profuse rectal bleeding, especially in children and young adults [4]. In these cases surgical resection is advised. In patients with peptic ulceration, treatment with $\mathrm{H}_{2}$-blockers or proton pump inhibitors should be prescribed first. A rectal Meckel's diverticulum may also be asymptomatic, as it was in our patient. We choose not to perform preventive resection or regular endoscopic screening, so long as the course is uneventful.

Endoscopy_UCTN_Code_CCL_1AD_2AJ

\section{N. K. H. de Boer, J. P. Kuyvenhoven}

Department of Gastroenterology and

Hepatology, Kennemer Gasthuis, Haarlem, The Netherlands

\section{References}

1 Ewell GH, Jackson RH. Aberrant gastric mucosa in the rectum with ulceration and hemorrhage. World J Surg 1939; 38: 641 -643

2 Garmendia M, Aparicio JR, Martínez J et al. Heterotopic gastric mucosa in the rectum. Gastrointest Endosc 2007; 65: 921

3 Stockman JM, Young VT, Jenkins AL. Duplication of the rectum containing gastric mucosa. JAMA 1960; 173: $1223-1225$

4 Forshaw JF, Dhia D, Cole S et al. Rectal Meckel's diverticulum: an unusual cause of rectal bleeding. Int J Colorectal Dis 2006; 21: $485-487$

\section{Bibliography}

DOI 10.1055/s-0029-1214696

Endoscopy 2009; 41: E258

(c) Georg Thieme Verlag KG Stuttgart · New York . ISSN 0013-726X

\section{Corresponding author}

\section{Nanne K. H. de Boer, MD, PhD}

Department of Gastroenterology and Hepatology Kennemer Gasthuis

PO Box 417

$2000 \mathrm{AK}$

Haarlem

The Netherlands

KHN.deBoer@vumc.nl 